\title{
Article \\ High-Throughput NanoBiT-Based Screening for Inhibitors of HIV-1 Vpu and Host BST-2 Protein Interaction
}

\author{
Boye Li $\mathrm{Li}^{1,2,3}$, Xiaoxiao Dong 1,3, Wenmei Zhang ${ }^{1,2,3}$, Tian Chen ${ }^{1,3}$, Boyang $\mathrm{Yu}^{1,3}$, Wenyue Zhao ${ }^{1,3}$, Yishu Yang ${ }^{1,3}$, \\ Xiaoli Wang ${ }^{1,3}$, Qin $\mathrm{Hu}^{1,3, *(1)}$ and Xiayan Wang ${ }^{1,2, *}$ \\ 1 The Faculty of Environment and Life, Beijing University of Technology, Beijing 100124, China; \\ liboye@emails.bjut.edu.cn (B.L.); dxx0119@emails.bjut.edu.cn (X.D.); \\ zwm1436271046@emails.bjut.edu.cn (W.Z.); chentian@emails.bjut.edu.cn (T.C.); \\ yuby@emails.bjut.edu.cn (B.Y.); clairezhaooo@163.com (W.Z.); yishu-y@bjut.edu.cn (Y.Y.); \\ wangxiaoli@bjut.edu.cn (X.W.) \\ 2 Center of Excellence for Environmental Safety and Biological Effects, Beijing Key Laboratory for Green \\ Catalysis and Separation, Department of Chemistry and Biology, Beijing University of Technology, \\ Beijing 100124, China \\ 3 Beijing International Science and Technology Cooperation Base of Antivirus Drug, Beijing University of \\ Technology, Beijing 100124, China \\ * Correspondence: hq07616@bjut.edu.cn (Q.H.); xiayanwang@bjut.edu.cn (X.W.)
}

check for updates

Citation: Li, B.; Dong, X.; Zhang, W.; Chen, T.; Yu, B.; Zhao, W.; Yang, Y.; Wang, X.; Hu, Q.; Wang, X. High-Throughput NanoBiT-Based Screening for Inhibitors of HIV-1 Vpu and Host BST-2 Protein Interaction. Int. J. Mol. Sci. 2021, 22, 9308. https://doi.org/10.3390/ijms 22179308

Academic Editor: Salvador Ventura

Received: 17 July 2021

Accepted: 25 August 2021

Published: 27 August 202

Publisher's Note: MDPI stays neutral with regard to jurisdictional claims in published maps and institutional affiliations.

Copyright: (c) 2021 by the authors. Licensee MDPI, Basel, Switzerland. This article is an open access article distributed under the terms and conditions of the Creative Commons Attribution (CC BY) license (https:// creativecommons.org/licenses/by/ $4.0 /)$.

\begin{abstract}
Bone marrow stromal cell antigen 2 (BST-2), also known as CD317 or tetherin, has been identified as a host restriction factor that suppresses the release of enveloped viruses from host cells by physically tethering viral particles to the cell surface; however, this host defense can be subverted by multiple viruses. For example, human immunodeficiency virus (HIV)-1 encodes a specific accessory protein, viral protein $\mathrm{U}(\mathrm{Vpu})$, to counteract BST-2 by binding to it and directing its lysosomal degradation. Thus, blocking the interaction between Vpu and BST-2 will provide a promising strategy for anti-HIV therapy. Here, we report a NanoLuc Binary Technology (NanoBiT)based high-throughput screening assay to detect inhibitors that disrupt the Vpu-BST-2 interaction. Out of more than 1000 compounds screened, four inhibitors were identified with strong activity at nontoxic concentrations. In subsequent cell-based BST-2 degradation assays, inhibitor Y-39983 HCl restored the cell-surface and total cellular level of BST-2 in the presence of Vpu. Furthermore, the Vpu-mediated enhancement of pesudotyped viral particle production was inhibited by $\mathrm{Y}-39983 \mathrm{HCl}$. Our findings indicate that our newly developed assay can be used for the discovery of potential antiviral molecules with novel mechanisms of action.
\end{abstract}

Keywords: HIV-1; Vpu; BST-2; NanoLuc Binary Technology; high-throughput screening assay

\section{Introduction}

Acquired immunodeficiency syndrome (AIDS) is a malignant infectious disease caused by human immunodeficiency virus (HIV), which causes severe destruction of immune function and eventually leads to opportunistic infections and tumors. Current antiretroviral drugs are often classified into six main classes: chemokine receptor antagonists, fusion inhibitors, nucleosides/non-nucleoside reverse transcriptase inhibitors, protease inhibitors, and integrase inhibitors. Although the advent and continuous optimization of combination antiretroviral therapy (cART) has significantly reduced the mortality and morbidity due to HIV infection [1,2], many challenges remain to be overcome, including incomplete adherence to ART, adverse effects, and drug resistance [3]. Therefore, there is still a major need for new antiviral interventions that target novel mechanisms of action.

The HIV-1 viral protein U (Vpu) protein is a type I transmembrane protein expressed in the late stage of the viral life cycle $[4,5]$ and a multifunctional accessory protein that facilitates viral egress. $\mathrm{Vpu}$ is found to counteract bone marrow stromal cell antigen 2 (BST-2) by downregulating its expression on the cell surface [6,7] and it also targets 
newly synthesized clusters of differentiation 4 (CD4) in the endoplasmic reticulum to prevent its trafficking to the plasma membrane and the unwanted formation of premature viral gp160/CD4 complex [8,9]. In addition, Vpu forms cation-selective ion channels or pores in lipid bilayers and possibly augments virion release [10]. In recent years, several new functions have been assigned to $\mathrm{Vpu}$, including the downregulation of $\mathrm{CD} 1 \mathrm{~d}$, major histocompatibility complex (MHC) molecules, NK, T- and B-cell antigen (NTB-A), and intercellular adhesion molecule 1 (ICAM-1) on the cell surface [11-14], deregulation of the nuclear factor kappa B (NF-kB) pathway, and modulation of DNA repair machinery [15]. Although an increasing number of novel targets have been identified, Vpu-BST-2 interaction remains the most active area in Vpu-related research.

BST-2 protein (also known as tetherin, CD317, or HM1.24) is a type II transmembrane protein with an N-terminal cytoplasmic domain and a C-terminal glycosyl-phosphatidylinositol (GPI) anchor. Due to its unique topology, BST-2 physically crosslinks the virions to the surface of infected cells, thereby suppressing the release of newly produced virus particles [16]. It exerts broad-spectrum activities against a variety of enveloped viruses and can be antagonized by different viral proteins, including HIV-1 Vpu $[17,18]$. The Vpu protein binds to BST-2 via interaction with the transmembrane (TM) region and induces BST-2 ubiquitination through an endosome-lysosome degradation pathway [19]. In addition to enhancing virus release, Vpu counteracts the role of BST-2 in interferon production and antibody-dependent cellular cytotoxicity [20,21]. Therefore, the Vpu-BST-2 interaction is a potential therapeutic target for the development of novel anti-HIV-1 treatments. In recent years, several agents have been identified [22-24], but no treatment has yet been approved.

In the present study, we used NanoLuc Binary Technology (NanoBiT) [25] to configure a cell-based Vpu-BST-2 binding assay for high-throughput small-molecule screening. We identified four inhibitors out of 1177 compounds from the immunology/inflammation compound library. They were further tested for activity on Vpu-induced BST-2 degradation and Vpu-mediated pseudotyped viral particle production.

\section{Results}

\subsection{Development of NanoBiT Assay for Detecting Vpu-BST-2 Interactions}

NanoBiT is a luciferase-based complementation reporter system that is widely used for the quantitative analysis of protein-protein interactions. The NanoBiT system is composed of a large (LgBiT, $18 \mathrm{kDa}$ ) and small BiT subunit (SmBiT, $1.3 \mathrm{kDa})$. In our study, Vpu and BST-2 proteins were fused to LgBiT and SmBiT, respectively, and co-expressed in HEK293T cells. When Vpu binds to BST-2, LgBiT and SmBiT subunits are brought together, resulting in the formation of NanoLuc luciferase (NLuc), which can be quantitatively measured and is applicable to high-throughput screening (Figure 1A). To optimize the orientation of LgBiT and SmBiT on Vpu and BST-2, respectively, we constructed eight pairs of Vpu and BST-2 containing vectors with either LgBiT or SmBiT fused to the N- or C-terminus. Following co-transfection into HEK293T cells, the luminescence signal was detected in all $\mathrm{Vpu} / \mathrm{BST}-2$ pairs and the highest luciferase activity was observed in CLV-NSB (Vpu with C-terminal LgBiT and BST-2 with N-terminal SmBiT), which generated a 95-fold increase over the corresponding control (Vpu with C-terminal LgBiT and HaloTag with SmBiT) as shown in Figure 1B. This combination was selected for the follow-up experiments. In addition, we detected the half-life of the luminescence signal and found that the signal of CLV-NSB increased in the first $2 \mathrm{~min}$ and decreased slightly afterward; it remained 100-fold higher than that of the control after $15 \mathrm{~min}$ (Figure 1C). This indicates that the luminescence signal was highly stable during the first $15 \mathrm{~min}$. 
A
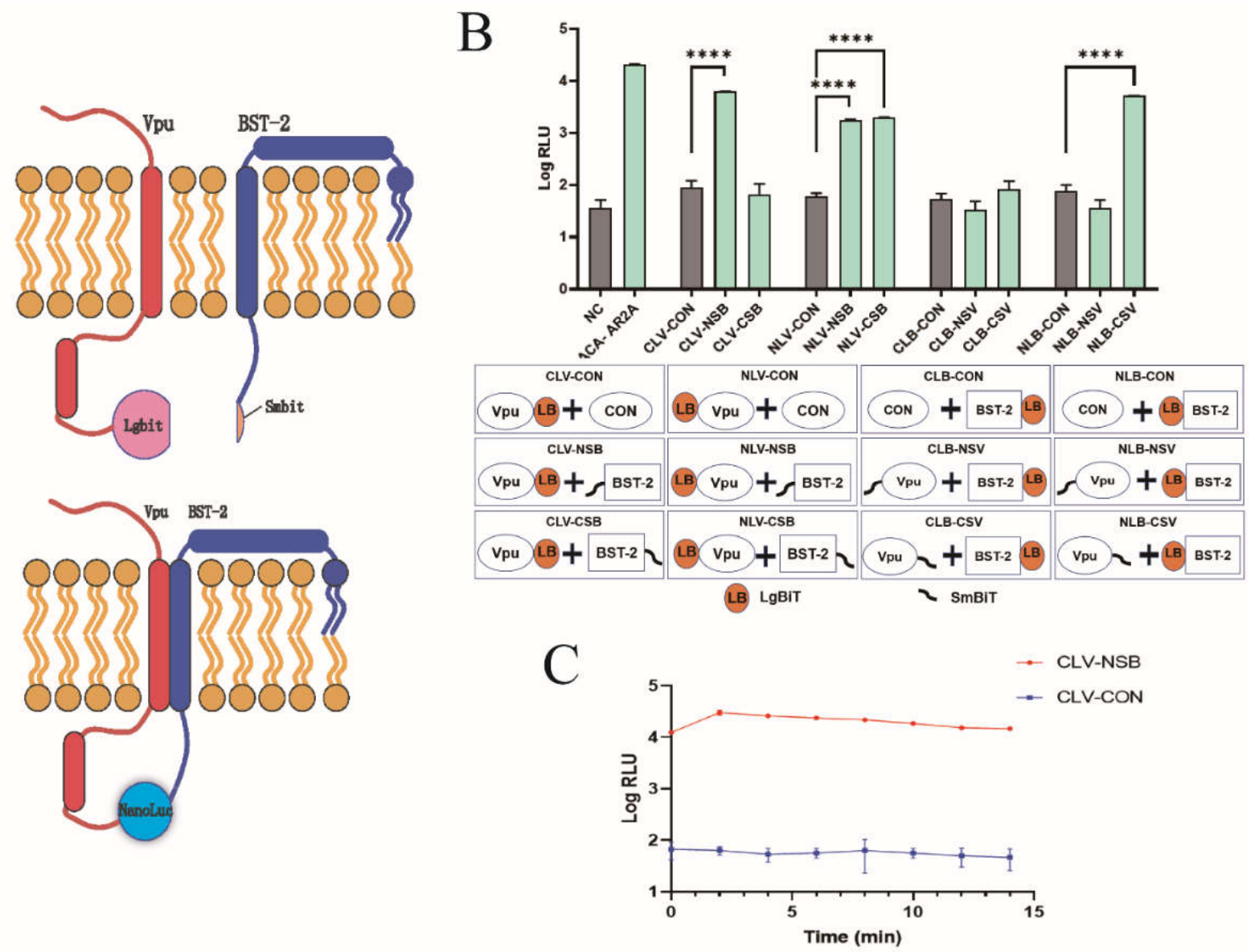

Figure 1. Development of the NanoBiT assay for detecting Vpu-BST-2 interaction. (A) Illustrations of the NanoBiT assay to monitor Vpu-BST-2 interaction. (B) Optimization of Vpu and BST-2 plasmid combinations. HEK293T cells were cotransfected with eight plasmid combinations as well as their respective controls. Two days later, the fluorescence values were measured to find the combination with the highest luciferase activity. ${ }^{* * *}, p<0.0001$ vs. control group. (NLB: pBiT1.1N-bst-2; NSB: pBiT2.1-N-bst-2; CLB: pBiT1.1-C-bst-2; CSB: pBiT2.1-C-bst-2; NLV: pBiT1.1-N-vpu; NSV: pBiT2.1-N-vpu; CLV: pBiT1.1-C-vpu; CSV: pBiT2.1-C-vpu; NC: negative control; CON: SmBiT vector control; ACA-AR2A: PRKACA: PRKAR2A pair was used as a constitutive positive control) (C) HEK293T cells were co-transfected with plasmids CLV and NSB. Two days later, after adding the substrate, the detection was carried out every 2 min to determine the optimal timepoint of NanoBiT assay. Values presented are the mean \pm standard deviation (SD) of three replicates. All experiments were performed five times.

\subsection{Identification of Inhibitors Targeting Vpu-BST-2 Interactions}

For the first round of screening, a total of 1177 compounds were initially screened for inhibitory activity on Vpu-BST-2 interactions, and 21 hits were identified with an inhibition rate greater than $85 \%$ (Figure $2 \mathrm{~A}$ ). In the confirmatory repeat screening, only four compounds (Y-39983 HCl, brazilin, tangeretin, and AS1517499) significantly inhibited the luminescence signal at $10 \mu \mathrm{mol} / \mathrm{L}$ (Figure 2B), and they were further tested in titrations to evaluate the cytotoxicity and activity of Vpu-BST-2 binding in parallel. The therapeutic indexes $\left(\mathrm{CC}_{50} / \mathrm{EC}_{50}\right)$ for Y-39983 HCl, brazilin, tangeretin, and AS1517499 were $>91,4.5$, $>14.8$, and 47, respectively (Figure 2C-F and Table S1), indicating an obvious binding inhibition efficacy and low cytotoxicity of compounds. 
A

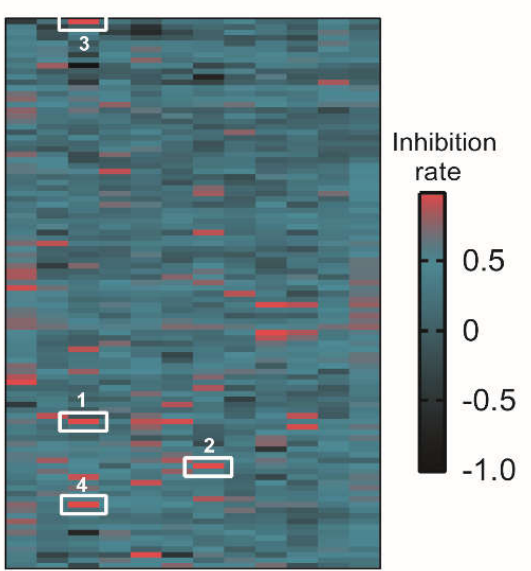

C

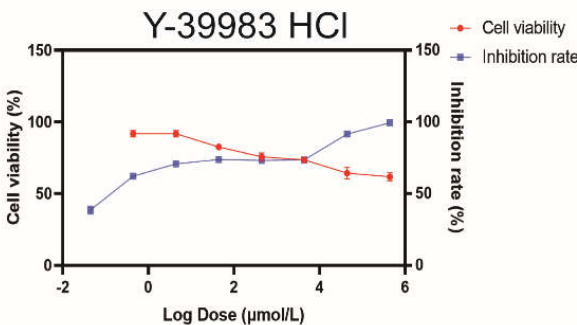

E

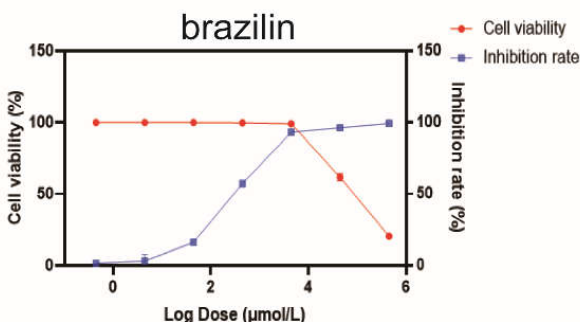

B

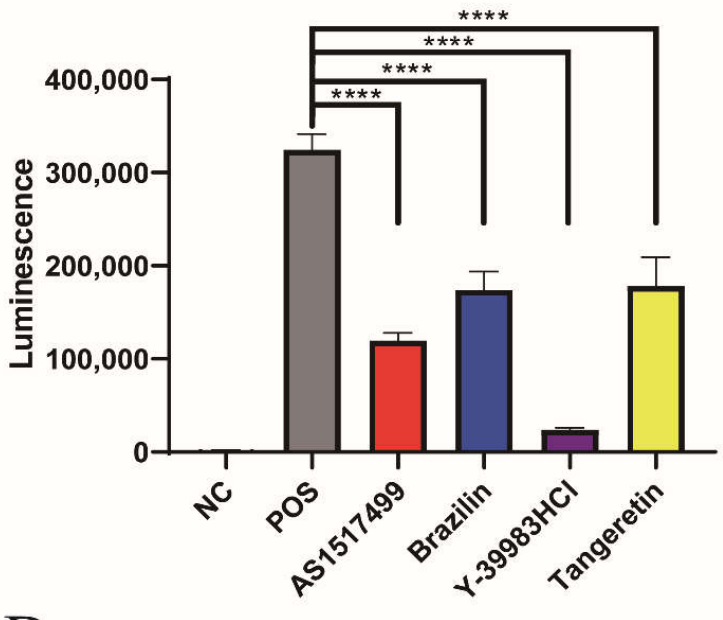

D

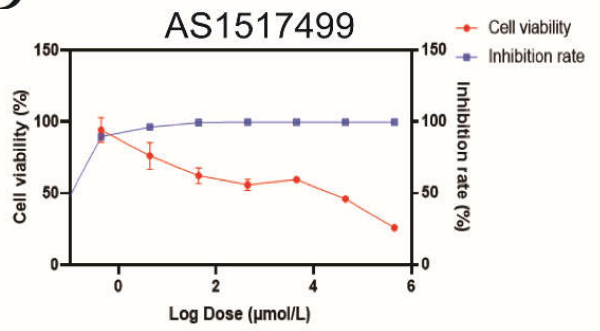

$\mathrm{F}$

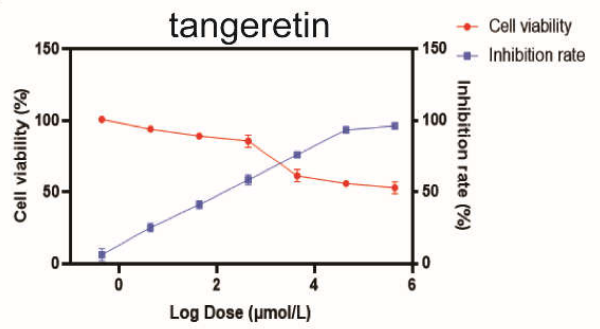

Figure 2. High-throughput compound screening by NanoBiT assay. (A) Composite heat map of 1177 compounds screened by NanoBiT assay. HEK293T Cells were co-transfected with plasmids CLV (Vpu with C-terminal LgBiT) and NSB (BST-2 with $\mathrm{N}$-terminal SmBiT). Compounds with a final concentration of $10 \mu \mathrm{mol} / \mathrm{L}$ were added $4 \mathrm{~h}$ later. The heat map is drawn from the calculated inhibition rate. AS1517499 (1), Y-39983 HCl (2), brazilin (3) and tangeretin (4) were labeled with white rectangles. (B) HEK293T cells were co-transfected with plasmids CLV and NSB and then treated with Y-39983 $\mathrm{HCl}$, brazilin, tangeretin, or AS1517499 $(10 \mu \mathrm{mol} / \mathrm{L})$, respectively. Luciferase activities were measured using a multilabel reader. ${ }^{* * *}, p<0.0001$ vs. postive control group. Dose-dependent curves of the inhibition rate and cell viability of HEK293T cells treated with (C) Y-39983 HCl, (D) AS1517499, (E) brazilin, or (F) tangeretin. Values presented are the mean \pm SD of three replicates.

\subsection{Protective Activity of Compounds against Vpu-Induced BST 2 Degradation}

To explore whether the disruption of Vpu and BST-2 interaction could reverse the Vpu-mediated downregulation of BST-2, HEK293T cells were transfected with CLV /NSB vectors and treated with Y-39983 HCl, brazilin, tangeretin, or AS1517499 at $10 \mu \mathrm{mol} / \mathrm{L}$ for $36 \mathrm{~h}$. The expression of total cellular BST-2 was imaged and measured using a highcontent imaging system, which showed that Vpu induced a remarkable downregulation of BST-2 (by 55\%) and that this effect was reversed by the addition of Y-39983 $\mathrm{HCl}$ and AS1517499 (Figure 3A). Similar trends were observed in Western blotting (Figure 3B). Since HEK293T cells were known as BST-2 negative cells, we also examined the endogenous BST-2 levels in Hela cells after transfection with Vpu vector (CLV). Consistent with the 
findings in HEK293T cells, the results of immunofluorescence analysis (Figure 3C) and Western blotting (Figure 3D) in Hela cells showed that Y-39983 HCl and AS1517499 restored the total cellular level of endogenous BST-2 in the presence of Vpu.

A
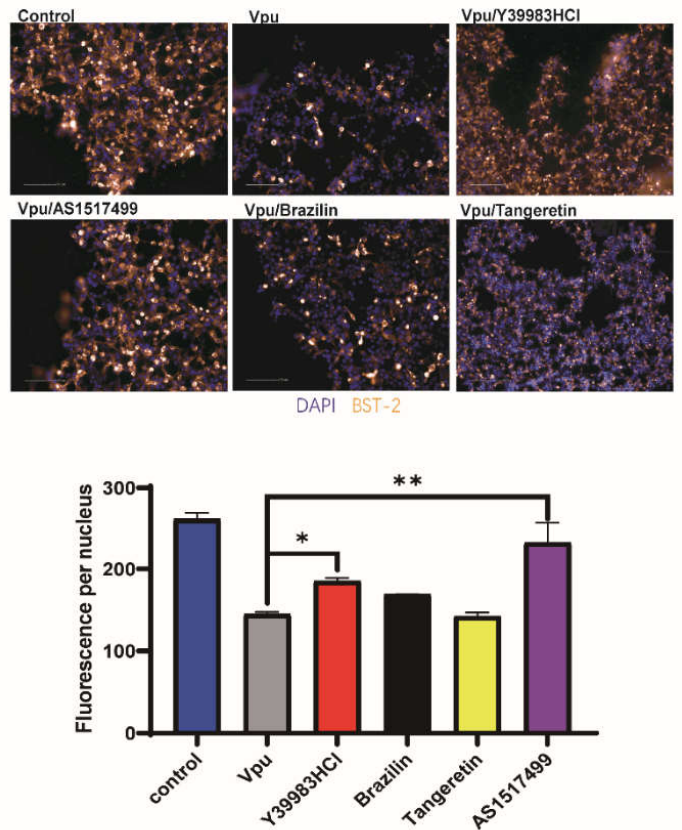

$\mathrm{C}$

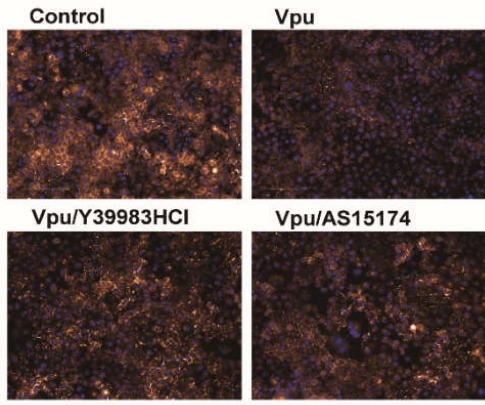

DAPI BST-2

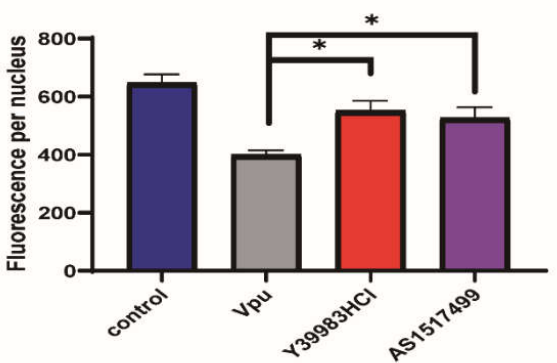

B

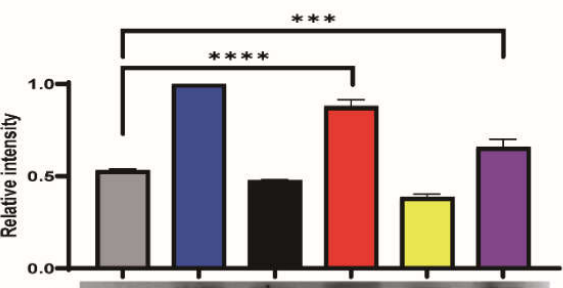

anti-BST-2$$
\text { anti- } \beta
$$

Vpu

Brazilin

Y-39983 HCI

Tangeretin

AS1517499

$\mathrm{D}$

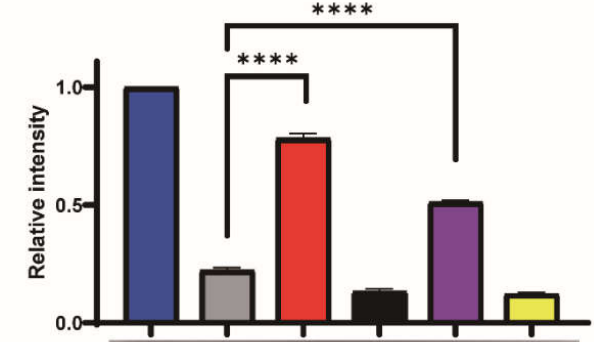

anti-BST-2

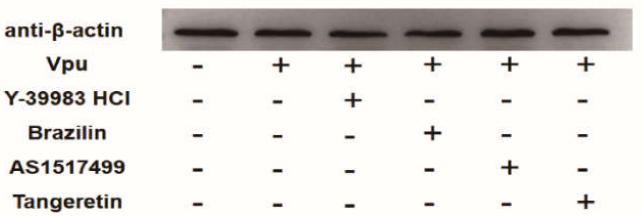

$\mathrm{E}$

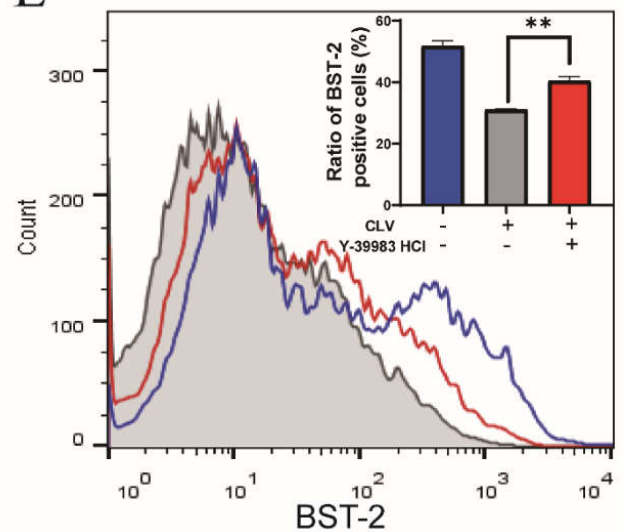

Figure 3. Compounds inhibited Vpu-mediated downregulation of BST-2. HEK293T cells were co-transfected with plasmids CLV (Vpu with C-terminal LgBiT) and NSB (BST-2 with N-terminal SmBiT), and treated with Y-39983 HCl, brazilin, tangeretin, or AS1517499 $(10 \mu \mathrm{mol} / \mathrm{L})$ for $36 \mathrm{~h}$. Total cellular expression of BST-2 was measured using a high-content imaging system (A) and Western blotting (B). (C) HeLa cells were transfected with CLV vector and treated with Y-39983 HCl, brazilin, tangeretin, or AS1517499 $(10 \mu \mathrm{mol} / \mathrm{L})$ for $36 \mathrm{~h}$. Total cellular expression of BST-2 was measured by high-content imaging analysis and Western blotting (D). (E) HEK293T cells were co-transfected with plasmids CLV and NSB, and treated with Y-39983 HCl, the cell-surface expression of BST-2 was detected by flow cytometry. ${ }^{*}, p<0.05 ;{ }^{* *}, p<0.01$; ${ }^{* * *}, p<0.001 ; * * * *, p<0.0001$ vs. Vpu group. Values presented are the mean $\pm \mathrm{SD}$ of three replicates. All experiments were performed thrice. 
Furthermore, we analyzed cell surface BST-2 levels by flow cytometry and found that only Y-39983 $\mathrm{HCl}$ upregulated the surface expression of BST-2 in the presence of Vpu (Figure 3E), whereas others had no effect on the surface BST-2 levels (Figure S2). These data suggest a protective role of $\mathrm{Y}-39983 \mathrm{HCl}$ against $\mathrm{Vpu}$ induced degradation of total cellular BST-2 as well as the cell surface BST-2.

\subsection{Antiviral Activity of Y-39983 HCl}

We evaluated the antiviral activity of $\mathrm{Y}-39983 \mathrm{HCl}$ on the production of new pseudotyped viral particles. HEK293T cells were co-transfected with the Vpu vector (CLV), BST-2 vector (NSB) and proviral vectors with defective vpu gene in the presence of Y-39983 HCl. After transfection, the Vpu expression was detected by Western blotting and showed an overexpression of Vpu in transfected HEK293T cells, and Y-39983 HCl did not affect the expression of Vpu protein (Figure 4A), indicating that the inhibition effect of $\mathrm{Y}-39983 \mathrm{HCl}$ on Vpu-BST-2 interaction was not mediated by affecting Vpu expression. Next, the envelope (Env)-pseudotyped virions were collected from the supernatant and tested using TZM-bl pseudovirus assay. TZM-bl is a Hela-derived cell line that expresses CD4, CCR5/CXCR5 to allow virus entry and contains a luciferase reporter gene under the control of an HIV Tat-responsive reporter. Over the years, the TZM-bl assay has been standardized as a classical method to quantify the infectious viral particle. The data showed a significant increase in the infectivity of new virions after Vpu overexpression, and the effect of Vpu was antagonized by Y-39983 HCl (Figure 4B). The virus stock was then titrated in TZM-bl cells to determine the $50 \%$ Tissue Culture Infective Dose $\left(\mathrm{TCID}_{50}\right)$ and we found that overexpression of Vpu promoted a higher production of new virions ( $\operatorname{TCID}_{50} 40,500 / \mathrm{mL}$ ) compared to the control ( TCID $_{50} 23,380 / \mathrm{mL}$ ), and treatment with Y-39983 HCl decreased virion release (TCID $5028,080 / \mathrm{mL}$ ). The inhibition of virion production by $\mathrm{Y}-39983 \mathrm{HCl}$ was further confirmed by the measurement of HIV-1 P24 Gag protein using enzyme-linked immunosorbent assay (ELISA; Figure 4C). In addition, we tested AS1517499 on virus production and found no antiviral activity, despite the fact that AS1517499 restored total cellular BST-2 level (Figure S3).

We also used CLV vector transfected Hela cells to produce envelope (Env)-pseudotyped virions and quantify virus via the TZM-bl luciferase reporter assay. We found that Y$39983 \mathrm{HCl}$ significantly reduced the increased infectivity of new virions induced by Vpu (Figure $4 \mathrm{D}$ ). The $\mathrm{TCID}_{50}$ assay also revealed that Vpu increased the production of new virions (TCID $5017,523 / \mathrm{mL}$ ) compared to the control (TCID $507015 / \mathrm{mL}$ ), and the effect of Vpu was antagonized by Y-39983 $\mathrm{HCl}\left(\mathrm{TCID}_{50} 8424 / \mathrm{mL}\right)$. We obtained a similar result from HIV capsid P24 protein ELISA assay (Figure 4E).

A

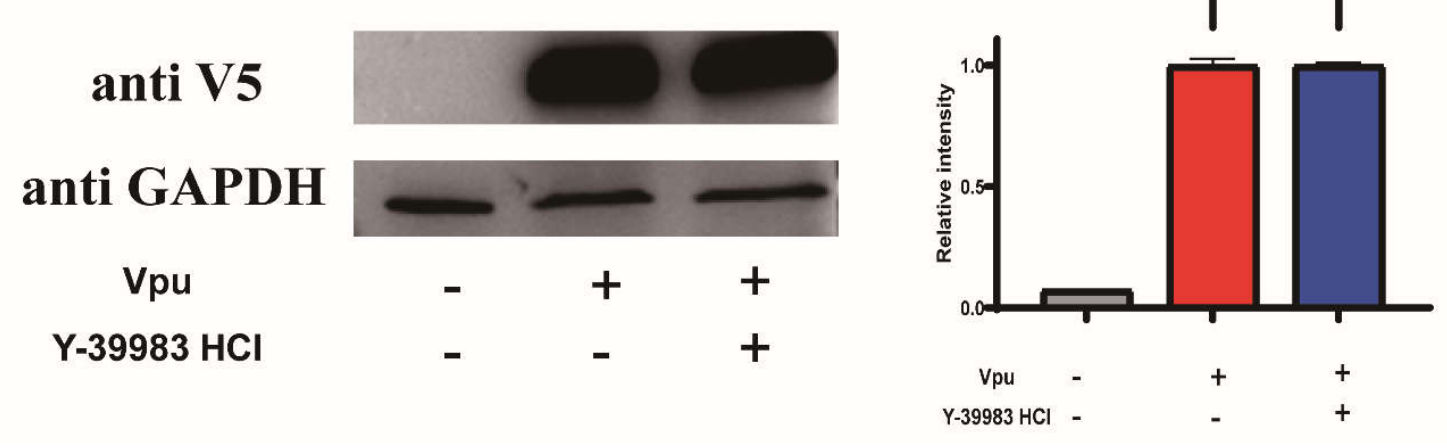

Figure 4. Cont. 
B

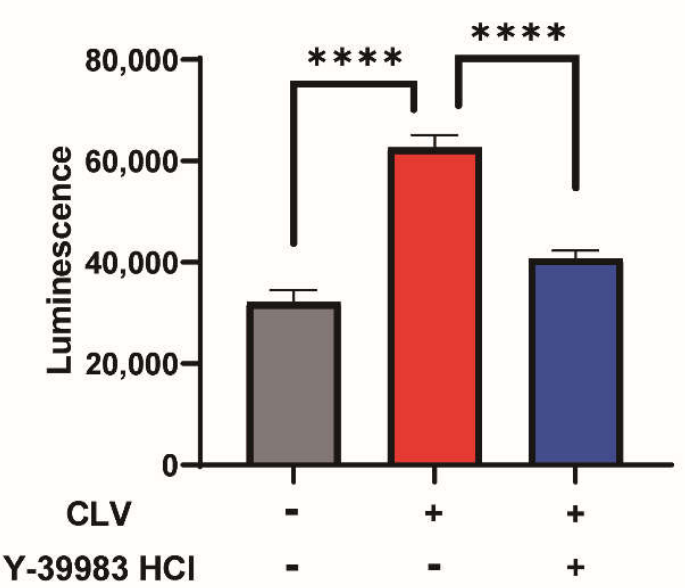

$\mathrm{D}$

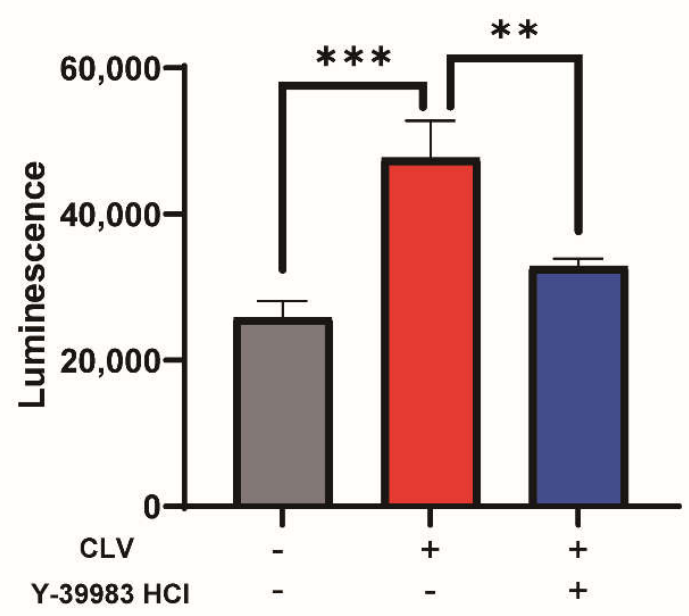

$\mathrm{C}$

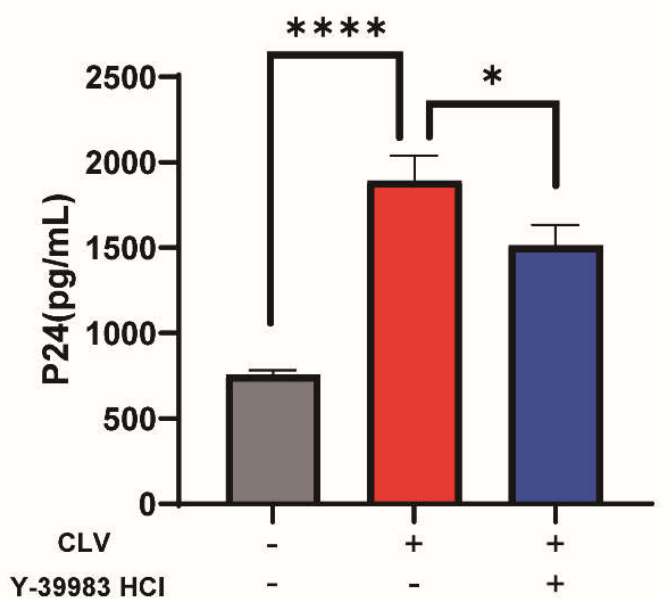

$\mathrm{E}$

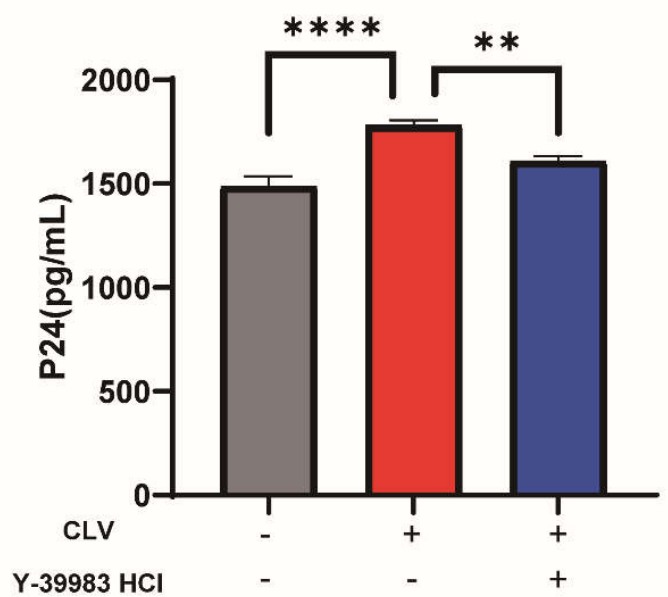

Figure 4. Y-39983 HCl reduced HIV-1 particle release. HEK293T cells were co-transfected with CLV-NSB plasmid or empty LgBiT vector (CLG)-NSB. Four hours after transfection, the cells were co-transfected with HIV Env expression plasmid pREJO4541.67 and backbone vector pSG3 $\Delta$ env in the presence of Y-39983 HCl. After 2 days, cells were lysed to detect Vpu expression by Western blotting (A). Env-pseudotyped virions were collected from the supernatant and used to infect TZM-bl cells. Two days after infection, the cells were lysed to evaluate luciferase activity using Bright-Glo Luciferase Assay System (B) and the supernatant was collected for p24 detection using ELISA assay (C). (D) HeLa cells were transfected with plasmid CLV or CLG. After $4 \mathrm{~h}$, cells were co-transfected with HIV Env expression plasmid pREJO4541.67 and backbone vector pSG3 $\Delta$ env in the presence of Y-39983 HCl. Virions were collected from the supernatant and quantitated using TZM-bl assay. The luciferase activity was measured using Bright-Glo Luciferase Assay System. The supernatant of TZM-bl culture were collected for HIV-1 p24 detection using ELISA assay (E). ns, Not Significant; ${ }^{*}, p<0.05 ;{ }^{* *}, p<0.01 ;{ }^{* * *}, p<0.001$; $* * * *, p<0.0001$ vs. Vpu group. Values presented are the mean \pm SD. All experiments were repeated thrice.

\section{Discussion}

In the present study, we developed a NanoBiT-based high-throughput screening assay to identify inhibitors of Vpu-BST-2 interactions. Out of 1177 compounds, we successfully identified four that blocked Vpu-BST-2 binding. Our data showed that one compound, Y-39983 HCl, significantly inhibited Vpu-mediated degradation of total cellular and cellsurface BST-2. Moreover, Y-39983 $\mathrm{HCl}$ reduced the production of newly synthesized viral particles via BST-2 restoration.

NanoBiT technology was used in our work to set up a real-time assay for monitoring Vpu-BST-2 interactions in HEK293T cells. The NanoBiT assay is based on structural complementation between the LgBiT, (156 amino acids) and SmBiT (11 amino acids) of NLuc [25]. The fragments by themselves are not active, but when protein pairs of interest interact with each other, both fragments are brought into close proximity and reconstitute the intact 
NLuc that can be quantitatively assessed. The assay was a powerful tool in characterizing intercellular protein-protein interactions in live cells $[25,26]$. To optimize it for the Vpu-BST2 interaction assay, we produced eight recombinant protein pairs to investigate whether the position and size of tags had an impact on luciferase signal. Numerous works [19,27-30] have identified that Vpu and BST-2 directly interact with each other via the transmembrane domain. Our data revealed that placing a tag at the N- and C-terminus of BST-2 and Vpu proteins, respectively, did not interfere with protein-protein binding, whereas a LgBiT tag fused to the C-terminus of BST-2 (CLB) showed a clear reduction in Vpu binding capacity, probably due to a change in protein localization. Protein pairs (CLV-NSB) that produced the highest luciferase signals were selected for subsequent experiments.

In previous studies, several compounds have been reported to protect BST-2 from Vpu-mediated degradation [23,24,31]; however, their mechanism of action is unclear. Here, using the NanoBiT-based Vpu-BST-2 interaction assay, we screened a total of 1177 small molecules and identified four compounds (Y-39983 HCl, brazilin, tangeretin, and AS1517499) with inhibitory activity against Vpu-BST-2 interaction. Y-39983 $\mathrm{HCl}$ is a selective Rho-associated protein kinase (ROCK) inhibitor [32,33], As1517499 is a signal transducer and activator of transcription 6 inhibitor, and tangeretin and brazilin are natural products with multiple functions. None of them had previously been reported to have anti-HIV activity. We also calculated the $\mathrm{IC}_{50}$ and $\mathrm{EC}_{50}$ to monitor the safety and efficacy of the compounds and found that all compounds exhibited dose-dependent activity and low cytotoxicity.

Next, we investigated whether inhibitors could inhibit the degradation of BST-2 induced by Vpu. As is known, HEK293T cells are BST-2 negative cells and Hela cells constitutively expressed BST-2 protein. We used both cell lines in our study and the results were consistent with those of previous studies [19,29], which showed that Vpu induced downregulation of cell-surface and total cellular expression of BST-2. It has been noted that Vpu-mediated BST-2 degradation varies depending on the cell type [28]. Vpu was shown to downregulate BST-2 at the cell surface as well as remove it from viral budding sites and prevent the recycling of BST-2 or de novo-synthesized BST-2 to the plasma membrane [34,35]. Although the degradation mechanisms remain controversial, the importance of Vpu-mediated surface downregulation of BST-2 in viral egress is welldefined [36]. In our study, with the addition of Y-39983 HCl and AS1517499, the total cellular levels of BST-2 were restored despite the presence of Vpu. However, only Y-39983 $\mathrm{HCl}$ upregulated the cell-surface expression of BST-2. Therefore, Y-39983 HCl may have the ability to prevent virus release via BST-2 restoration on the cell surface and was selected for further evaluation.

Our results also suggest that the infectivity of HIV-1 virus can be inhibited by Y$39983 \mathrm{HCl}$. HIV-1 Env-pseudotyped viruses were produced by transfection of HeLa and HEK293T cells with proviral DNA constructs (with defects in the vpu gene) and assessed using a TZM-bl assay. This assay is a quantitative method of measuring infectious viruses by a Tat-responsive Luc reporter and is widely used as a standardized HIV-1 neutralization assay $[37,38]$. Viruses were titrated on TZM-bl cells to calculate the viral titer $\left(\mathrm{TCID}_{50} / \mathrm{mL}\right.$ ) [39] and HIV-1 P24 antigen capture assay, a long-established method for detecting HIV-1 virus replication, was also used for virus quantitation [40,41]. Using TZM-bl cells, we found that overexpression of Vpu led to an increase in the infectivity of the released virus and the data are consistent with previous reports that demonstrated that Vpu promoted viral release by antagonizing BST-2 [14,31,42]. With the addition of Y-39983 $\mathrm{HCl}$, virus production was significantly inhibited, indicating that disruption of Vpu-BST-2 interaction and protection of BST-2 expression on cell surface contributed to the antiviral activity of Y-39983 HCl. 


\section{Materials and Methods}

\subsection{Cell Culture and Transfection}

HEK293T and HeLa cells were obtained from the American Type Culture Collection (ATCC, Rockville, MD, USA), and TZM-bl cells were obtained from the National Institutes of Health [NIH] AIDS Research and Reference Reagent Program. TZM-bl (JC, 53bl-13) cell line is a genetically modified HeLa cell line that expresses CD4 and CCR5/CXCR4 and contains the firefly luciferase and the LacZ reporter gene under the control of a Tatresponsive reporter for the detection of Tat-dependent luciferase and $\beta$-galactosidase activities after Env-pseudo typed viral infection. All cells were cultured in Dulbecco's modified Eagle medium (Gibco, Grand Island, NY, USA) supplemented with 10\% fetal bovine serum (FBS) (Gibco) and penicillin-streptomycin (Gibco). HEK293T and TZMbl cells were transfected using Lipofectamine ${ }^{\mathrm{TM}} 3000$ Transfection Reagent (Invitrogen, Carlsbad, CA, USA) according to the manufacturer's instructions.

\subsection{Plasmid and Compound Library}

The cloning vectors used to create $\mathrm{LgBiT}$ and $\mathrm{SmBiT}$ protein fusions were provided in the Nano-Glo ${ }^{\circledR}$ Live Cell Assay System (Promega, Madison, WI, USA). The vpu and BST-2 genes were subcloned into pBiT1.1-C [TK/LgBiT], pBiT2.1-C [TK/SmBiT], pBiT1.1-N [TK/LgBiT], and pBiT2.1-N [TK/SmBiT] vectors at the XhoI and NheI sites, respectively, to generate the fusion protein expression vector. A library of 1117 bioactive compounds was obtained from Selleck Chemicals (L4100 and L2000; Houston, TX, USA). Y-39983 HCl (S7935), brazilin (S9428), tangeretin (S2363), and AS1517499 (S8685) were also obtained from Selleck Chemicals. The structure of 4 compounds is shown in Figure S1.

\subsection{Western Blotting and Antibodies}

HEK293T and HeLa cells were lysed on ice for $15 \mathrm{~min}$ in $100 \mu \mathrm{L}$ radioimmunoprecipitation assay lysis buffer (Solarbio, Beijing, China) supplemented with a protease inhibitor cocktail (Promega). The protein concentration of lysates was determined using a bicinchoninic acid protein assay kit (Solarbio), and equal amounts of cellular proteins were suspended in NuPAGE LDS sample buffer (Invitrogen) and NuPAGE ${ }^{\mathrm{TM}}$ sample reducing buffer (Invitrogen), followed by boiling for $5 \mathrm{~min}$ at $95^{\circ} \mathrm{C}$. Proteins were then separated on $12 \%$ Mini-PROTEAN TGX gels (Bio-Rad, USA) and transferred onto polyvinylidene difluoride membranes. The membranes were then blocked with $5 \%$ non-fat milk in phosphatebuffered saline (PBS) containing 0.01\% Tween-20 and blotted with rabbit anti-human BST-2 (Abcam, UK) or rabbit anti-human $\beta$-actin antibodies (Abcam) overnight at $4{ }^{\circ} \mathrm{C}$. The express of V5 tagged Vpu protein was detected by mouse anti-V5 antibody (Thermo). The membranes were further incubated with horseradish peroxidase-labeled goat antirabbit secondary antibodies or goat anti-mouse secondary antibodies and detected using enhanced chemiluminescence. The protein bands were imaged using a Tanon 5200 multiimaging system (Tanon Science\& Technology Co., Ltd., Shanghai, China).

\subsection{Flow Cytometry}

For cell-surface staining, HEK293T cells were collected and stained with PE antihuman CD317 (BioLegend, CA, USA) at room temperature for $30 \mathrm{~min}$. Cells were then washed and resuspended in PBS $+1 \%$ FBS for FACS analysis using a BD FACS Calibur ${ }^{\mathrm{TM}}$ flow cytometer (BD Bioscience, San Diego, CA, USA). The data were analyzed using the FlowJo software V10 (Tree Star, Ashland, OR, USA).

\subsection{NanoBiT Assay}

HEK293T cells were plated in 96-well plates at a concentration of $1 \times 10^{5}$ cells $/ \mathrm{mL}$ and co-transfected with the plasmids encoding the Vpu and BST-2 fusion proteins at a ratio of 1:1. The empty vector (HaloTag with SmBiT) was used as a negative control. At $12 \mathrm{~h}$ post-transfection, the cells were treated with bioactive compounds (L2000 and L4100; Selleck Chemicals) at a final concentration of $10 \mu \mathrm{mol} / \mathrm{L}$ and incubated for $24 \mathrm{~h}$. 
Then, Nano-Glo Live Cell Substrate (N2012; Promega) was added and luminescence was measured using an EnSpire Multilabel Plate Reader 2300 (PerkinElmer, Waltham, MA, USA). The inhibition rate on Vpu-BST-2 binding was calculated as, inhibition rate $(\%)=$ $(1-$ experimental OD value/control OD value $) \times 100 \%$. A heat map of the inhibition rate of all compounds was generated using GraphPad Prism 8 software (GraphPad Software Inc., San Diego, CA, USA).

\subsection{Virus Release and Infectivity Analysis}

HEK293T and HeLa cells were seeded in 6-well plates. HEK293T cells were transfected with NSB and CLV plasmids containing the bst-2 and vpu genes. The empty large BiT vector (CLG) was used as a negative control. HeLa cells were transfected with CLV or CLG plasmids. Four hours after transfection, the cells were co-transfected with HIV Env expression plasmid pREJO4541.67 (NIH\#11035) and backbone vector pSG3 $\Delta$ env (NIH\#11051; NIH AIDS Research and Reference Reagent Program) in the presence of Y-39983 HCl. After $2 \mathrm{~d}$, recombinant viruses were collected from the supernatants. Then, TZM-bl cells were seeded in a 96-well plate in medium containing $10 \mu \mathrm{g} / \mathrm{mL}$ DEAE-dextran (Sigma) and infected with the Env-pseudotyped virus. Two days after infection, the supernatant was collected to quantify P24 level by HIV-1 p24/Capsid Protein p24 ELISA Kit (SinoBiological) following the manufacturer's instruction. Additionally, the cells were lysed to evaluate luciferase activity using the Bright-Glo Luciferase Assay System (Promega). To calculate the $50 \%$ tissue culture infective dose $\left(\mathrm{TCID}_{50}\right)$ of the Env-pseudotyped virus, TZM-bl cell were infected with serial 3-fold dilutions of the Env-pseudotyped virus (starting at 1: 10 dilutions). Two days after infection, cells were lysed to evaluate luciferase activity using the Bright-Glo Luciferase Assay System, TCID $_{50}$ was calculated using the Reed-Muench method $[43,44]$.

\subsection{Immunofluorescence}

HEK293T and HeLa cells were seeded in 6-well plates. HEK293T cells were transfected with NSB and CLV plasmids containing the bst-2 and vpu gene. The empty large BiT vector (CLG) was used as a negative control. HeLa cells were transfected with CLV or CLG plasmids. Twenty-four hours after transfection, the cells were fixed with $4 \%$ paraformaldehyde and permeabilized with $0.5 \%$ Triton X-100. After blocking with normal goat serum (Solarbio), the cells were stained with rabbit anti-human BST-2 antibody for $2 \mathrm{~h}$ at $37^{\circ} \mathrm{C}$ and then incubated with Alexa Fluor 594-conjugated goat anti-rabbit secondary antibody. The cells were then incubated with DAPI solution (Solarbio) for $10 \mathrm{~min}$ at room temperature. Immunofluorescence was imaged using the Operetta high-content imaging system (PerkinElmer) and quantitatively analyzed using the Harmony software (PerkinElmer). The mean fluorescence was calculated as follows: fluorescence per nucleus = fluorescence per well/cell number (DAPI positive cells).

\subsection{Cell Viability}

Cell viability was assessed using Cell Counting Kit-8 (CCK-8; Dojindo Molecular Technologies Inc., Tokyo, Japan). Briefly, HEK293T cells were treated with a serial dilution of Y-39983 HCl, brazilin, tangeretin, or AS1517499 ranging from 0.4 to $50 \mu \mathrm{mol} / \mathrm{L}$. Fortyeight hours after treatment, the cells were incubated with CCK-8 solution and absorbance was measured at $450 \mathrm{~nm}$ using an EnSpire Multilabel Plate Reader 2300 (PerkinElmer), and the relative cell viability was calculated as follows: cell viability $(\%)=$ experimental OD value/control OD value $\times 100 \%$.

\subsection{Statistical Analysis}

Data are presented as the mean \pm standard deviation, as indicated in the figure legends. Statistical analysis was performed by one-way analysis of variance using GraphPad Prism 8 software (GraphPad Software Inc., San Diego, CA, USA). Differences between groups were considered significant at $p<0.05$. 


\section{Conclusions}

Using NanoBiT technology, we developed a high-throughput screening assay to monitor Vpu-BST-2 interactions in live cells. We identified four compounds with inhibitory activity against the Vpu-BST-2 interaction. We further revealed that one compound, Y-39983 $\mathrm{HCl}$ restored BST-2 expression on the cell surface and inhibited viral particle production. Therefore, our study suggests a new application of NanoBiT technology in the development of novel antiviral drugs.

Supplementary Materials: The following are available online at https:/ / www.mdpi.com/article/10 .3390/ijms22179308/s1.

Author Contributions: Conceived and designed the experiments: B.L., Q.H., X.W. (Xiayan Wang) Performed the experiments: B.L., T.C., B.Y., X.W. (Xiaoli Wang), W.Z. (Wenyue Zhao), Y.Y. Analyzed the data: X.D., W.Z. (Wenmei Zhang), X.W. (Xiaoli Wang); Wrote the paper: Q.H., B.L. All authors have read and agreed to the published version of the manuscript.

Funding: This research was funded by the National Natural Science Foundation of China, grant number $(81760783,21625501,21936001)$ and Beijing Outstanding Young Scientist Program, grant number (BJJWZYJH01201910005017).

Acknowledgments: We thank the NIH AIDS Reagent Program for HIV-1 proviral plasmids and TZM-bl cells used in this study.

Conflicts of Interest: The authors declare that they have no known competing financial interests or personal relationships that could have appeared to influence the work reported in this paper.

\section{References}

1. Cohen, M.S.; Chen, Y.Q.; McCauley, M.; Gamble, T.; Hosseinipour, M.C.; Kumarasamy, N.; Hakim, J.G.; Kumwenda, J.; Grinsztejn, B.; Pilotto, J.H.; et al. Prevention of HIV-1 Infection with Early Antiretroviral Therapy. N. Engl. J. Med. 2011, 365, 493-505. [CrossRef]

2. Webb, E.L.; Ekii, A.O.; Pala, P. Epidemiology and immunology of helminth-HIV interactions. Curr. Opin. Hiv Aids. 2012, 7, 245-253. [CrossRef]

3. Mehellou, Y.; Clercq, E.D. Twenty-six years of anti-HIV drug discovery: Where do we stand and where do we go? J. Med. Chem. 2010, 53, 521-538. [CrossRef]

4. Cohen, E.A.; Terwilliger, E.F.; Sodroski, J.G.; Haseltine, W.A. Identification of a protein encoded by the vpu gene of HIV-1. Nature 1988, 334, 532-534. [CrossRef]

5. Magadán, J.G.; Bonifacino, J.S. Transmembrane Domain Determinants of CD4 Downregulation by HIV-1 Vpu. J. Virol. 2011, 86, 757-772. [CrossRef]

6. Jouvenet, N.; Neil, S.; Zhadina, M.; Zang, T.; Kratovac, Z.; Lee, Y.; McNatt, M.; Hatziioannou, T.; Bieniasz, P.D. Broad-Spectrum Inhibition of Retroviral and Filoviral Particle Release by Tetherin. J. Virol. 2009, 83, 1837-1844. [CrossRef]

7. Van Damme, N.; Goff, D.; Katsura, C.; Jorgenson, R.L.; Mitchell, R.; Johnson, M.C.; Stephens, E.B.; Guatelli, J. The InterferonInduced Protein BST-2 Restricts HIV-1 Release and Is Downregulated from the Cell Surface by the Viral Vpu Protein. Cell Host Microbe 2008, 3, 245-252. [CrossRef] [PubMed]

8. Schubert, U.; Henklein, P.; Boldyreff, B.; Wingender, E.; Strebel, K.; Porstmann, T. The Human Immunodeficiency Virus Type 1 Encoded Vpu Protein is Phosphorylated by Casein Kinase-2 (CK-2) at Positions Ser52 and Ser56 within a Predicted $\alpha$-Helix-Turn$\alpha$-Helix-Motif. J. Mol. Biol. 1994, 236, 16-25. [CrossRef] [PubMed]

9. Margottin, F.; Bour, S.; Durand, H.; Selig, L.; Benichou, S.; Richard, V.; Thomas, D.; Strebel, K.; Benarous, R. A Novel Human WD Protein, h- $\beta$ TrCP, that Interacts with HIV-1 Vpu Connects CD4 to the ER Degradation Pathway through an F-Box Motif. Mol. Cell 1998, 1, 565-574. [CrossRef]

10. Greiner, T.; Bolduan, S.; Hertel, B.; Groß, C.; Hamacher, K.; Schubert, U.; Moroni, A.; Thiel, G. Ion Channel Activity of Vpu Proteins Is Conserved throughout Evolution of HIV-1 and SIV. Viruses 2016, 8, 325. [CrossRef] [PubMed]

11. Apps, R.; Del Prete, G.Q.; Chatterjee, P.; Lara, A.; Brumme, Z.L.; Brockman, M.A.; Neil, S.; Pickering, S.; Schneider, D.K.; Piechocka-Trocha, A.; et al. HIV-1 Vpu Mediates HLA-C Downregulation. Cell Host Microbe 2016, 19, 686-695. [CrossRef]

12. Shah, A.H.; Sowrirajan, B.; Davis, Z.B.; Ward, J.; Campbell, E.M.; Planelles, V.; Barker, E. Degranulation of Natural Killer Cells Following Interaction with HIV-1-Infected Cells Is Hindered by Downmodulation of NTB-A by Vpu. Cell Host Microbe 2010, 8 , 397-409. [CrossRef]

13. Matheson, N.J.; Sumner, J.; Wals, K.; Rapiteanu, R.; Weekes, M.P.; Vigan, R.; Weinelt, J.; Schindler, M.; Antrobus, R.; Costa, A.S.; et al. Cell Surface Proteomic Map of HIV Infection Reveals Antagonism of Amino Acid Metabolism by Vpu and Nef. Cell Host Microbe 2015, 18, 409-423. [CrossRef] 
14. Moll, M.; Andersson, S.K.; Smed-Sörensen, A.; Sandberg, J.K. Inhibition of lipid antigen presentation in dendritic cells by HIV-1 Vpu interference with CD1d recycling from endosomal compartments. Blood 2010, 116, 1876-1884. [CrossRef] [PubMed]

15. Volcic, M.; Sparrer, K.M.J.; Koepke, L.; Hotter, D.; Sauter, D.; Stürzel, C.M.; Scherer, M.; Stamminger, T.; Hofmann, T.G.; Arhel, N.J.; et al. Vpu modulates DNA repair to suppress innate sensing and hyper-integration of HIV-1. Nat. Microbiol. 2020, 5, $1247-1261$. [CrossRef]

16. Venkatesh, S.; Bieniasz, P.D. Mechanism of HIV-1 Virion Entrapment by Tetherin. PLOS Pathog. 2013, 9, e1003483. [CrossRef] [PubMed]

17. Neil, S.; Zang, T.; Bieniasz, P.D. Tetherin inhibits retrovirus release and is antagonized by HIV-1 Vpu. Nature 2008, 451, 425-430. [CrossRef] [PubMed]

18. Daniel, S. Counteraction of the multifunctional restriction factor tetherin. Front. Microbiol. 2014, 5, 163. [CrossRef]

19. Douglas, J.L.; Viswanathan, K.; McCarroll, M.N.; Gustin, J.K.; Fruh, K.; Moses, A.V. Vpu Directs the Degradation of the Human Immunodeficiency Virus Restriction Factor BST-2/Tetherin via a beta TrCP-Dependent Mechanism. J. Virol. 2009, 83, 7931-7947. [CrossRef]

20. Alvarez, R.A.; Hamlin, R.E.; Monroe, A.; Moldt, B.; Hotta, M.T.; Caprio, G.R.; Fierer, D.; Simon, V.; Chen, B.K. HIV-1 Vpu Antagonism of Tetherin Inhibits Antibody-Dependent Cellular Cytotoxic Responses by Natural Killer Cells. J. Virol. 2014, 88, 6031-6046. [CrossRef]

21. Bego, M.G.; Édouard, C.; Nick, A.; Johanne, M.; Winfried, W.; Cohen, é.; Ross, S.R.J.P.P. Vpu Exploits the Cross-Talk between BST2 and the ILT7 Receptor to Suppress Anti-HIV-1 Responses by Plasmacytoid Dendritic Cells. PLoS Pathog. 2015, 11, e1005024. [CrossRef] [PubMed]

22. Pang, X.; Hu, S.; Li, J.; Xu, F.; Mei, S.; Zhou, J.; Cen, S.; Jin, Q.; Guo, F. Identification of novel key amino acids at the interface of the transmembrane domains of human BST-2 and HIV-1 Vpu. Retrovirology 2013, 10, 84. [CrossRef] [PubMed]

23. Zhang, Q.; Liu, Z.; Mi, Z.; Li, X.; Jia, P.; Zhou, J.; Yin, X.; You, X.; Yu, L.; Guo, F.; et al. High-throughput assay to identify inhibitors of Vpu-mediated down-regulation of cell surface BST-2. Antivir. Res. 2011, 91, 321-329. [CrossRef] [PubMed]

24. Zhang, Q.; Mi, Z.; Huang, Y.; Ma, L.; Ding, J.; Wang, J.; Zhang, Y.; Chen, Y.; Zhou, J.; Guo, F.; et al. 2-thio-6-azauridine inhibits Vpu mediated BST-2 degradation. Retrovirology 2016, 13, 1-14. [CrossRef] [PubMed]

25. Dixon, A.S.; Schwinn, M.K.; Hall, M.P.; Zimmerman, K.; Otto, P.; Lubben, T.H.; Butler, B.L.; Binkowski, B.F.; Machleidt, T.; Kirkland, T.A.; et al. NanoLuc Complementation Reporter Optimized for Accurate Measurement of Protein Interactions in Cells. ACS Chem. Biol. 2015, 11, 400-408. [CrossRef] [PubMed]

26. Wiertelak, W.; Sosicka, P.; Olczak, M.; Maszczak-Seneczko, D. Analysis of homologous and heterologous interactions between UDP-galactose transporter and beta-1,4-galactosyltransferase 1 using NanoBiT. Anal. Biochem. 2020, 593, 113599. [CrossRef]

27. Andrew, A.J.; Miyagi, E.; Strebel, K. Differential Effects of Human Immunodeficiency Virus Type 1 Vpu on the Stability of BST-2/Tetherin. J. Virol. 2010, 85, 2611-2619. [CrossRef] [PubMed]

28. Dube, M.; Paquay, C.; Roy, B.B.; Bego, M.G.; Mercier, J.; Cohen, A. HIV-1 Vpu Antagonizes BST-2 by Interfering Mainly with the Trafficking of Newly Synthesized BST-2 to the Cell Surface. Traffic 2011, 12, 1714-1729. [CrossRef] [PubMed]

29. Iwabu, Y.; Fujita, H.; Kinomoto, M.; Kaneko, K.; Ishizaka, Y.; Tanaka, Y.; Sata, T.; Tokunaga, K. HIV-1 Accessory Protein Vpu Internalizes Cell-surface BST-2/Tetherin through Transmembrane Interactions Leading to Lysosomes. J. Biol. Chem. 2009, 284, 35060-35072. [CrossRef]

30. Kobayashi, T.; Ode, H.; Yoshida, T.; Sato, K.; Gee, P.; Yamamoto, S.P.; Ebina, H.; Strebel, K.; Sato, H.; Koyanagi, Y. Identification of Amino Acids in the Human Tetherin Transmembrane Domain Responsible for HIV-1 Vpu Interaction and Susceptibility. J. Virol. 2010, 85, 932-945. [CrossRef]

31. Mi, Z.; Ding, J.; Zhang, Q.; Zhao, J.; Ma, L.; Yu, H.; Liu, Z.; Shan, G.; Li, X.; Zhou, J.; et al. A small molecule compound IMB-LA inhibits HIV-1 infection by preventing viral Vpu from antagonizing the host restriction factor BST-2. Sci. Rep. 2015, 5, 18499. [CrossRef]

32. Ramachandran, C.; Patil, R.; Combrink, K.; Sharif, N.; Srinivas, S. Rho-Rho kinase pathway in the actomyosin contraction and cell-matrix adhesion in immortalized human trabecular meshwork cells. Mol. Vis. 2011, 17, 1877-1890. [PubMed]

33. Gao, C.; Huang, L.; Long, Y.; Zheng, J.; Yang, J.; Pu, S.; Xie, L. Y-39983, a Selective Rho-Kinase Inhibitor, Attenuates Experimental Autoimmune Encephalomyelitis via Inhibition of Demyelination. Neuroimmunomodulation 2013, 20, 334-340. [CrossRef] [PubMed]

34. Dubé, M.; Roy, B.B.; Guiot-Guillain, P.; Binette, J.; Mercier, J.; Chiasson, A.; Cohen, É.; Luban, J.J.P.P. Antagonism of Tetherin Restriction of HIV-1 Release by Vpu Involves Binding and Sequestration of the Restriction Factor in a Perinuclear Compartment. PLoS Pathog. 2010, 6, e1000856. [CrossRef] [PubMed]

35. Schmidt, S.; Fritz, J.V.; Bitzegeio, J.; Fackler, O.T.; Keppler, O.T. HIV-1 Vpu Blocks Recycling and Biosynthetic Transport of the Intrinsic Immunity Factor CD317/Tetherin To Overcome the Virion Release Restriction. mBio 2011, 2, e00036-11. [CrossRef] [PubMed]

36. Skasko, M.; Tokarev, A.; Chen, C.-C.; Fischer, W.B.; Pillai, S.K.; Guatelli, J. BST-2 is rapidly down-regulated from the cell surface by the HIV-1 protein Vpu: Evidence for a post-ER mechanism of Vpu-action. Virology 2011, 411, 65-77. [CrossRef] [PubMed]

37. Todd, C.A.; Greene, K.M.; Yu, X.; Ozaki, D.A.; Gao, H.; Huang, Y.; Wang, M.; Li, G.; Brown, R.; Wood, B.; et al. Development and implementation of an international proficiency testing program for a neutralizing antibody assay for HIV-1 in TZM-bl cells. $J$. Immunol. Methods 2012, 375, 57-67. [CrossRef] [PubMed] 
38. Sarzotti-Kelsoe, M.; Bailer, R.T.; Turk, E.; Lin, C.-L.; Bilska, M.; Greene, K.M.; Gao, H.; Todd, C.A.; Ozaki, D.A.; Seaman, M.S.; et al. Optimization and validation of the TZM-bl assay for standardized assessments of neutralizing antibodies against HIV-1. J. Immunol. Methods 2013, 409, 131-146. [CrossRef] [PubMed]

39. Lei, C.; Yang, J.; Hu, J.; Sun, X. On the Calculation of TCID50 for Quantitation of Virus Infectivity. Virol. Sin. 2020, 36, 141-144. [CrossRef]

40. Speers, D.; Phillips, P.; Dyer, J. Combination Assay Detecting both Human Immunodeficiency Virus (HIV) p24 Antigen and Anti-HIV Antibodies Opens a Second Diagnostic Window. J. Clin. Microbiol. 2005, 43, 5397-5399. [CrossRef]

41. Fiebig, E.W.; Wright, D.J.; Rawal, B.D.; Garrett, P.E.; Busch, M.P.J.A. Dynamics of HIV viremia and antibody seroconversion in plasma donors: Implications for diagnosis and staging of primary HIV infection. AIDS 2003, 17, 1871-1879. [CrossRef] [PubMed]

42. Mwimanzi, P.; Tietjen, I.; Miller, S.C.; Shahid, A.; Cobarrubias, K.; Kinloch, N.N.; Baraki, B.; Richard, J.; Finzi, A.; Fedida, D.; et al. Novel Acylguanidine-Based Inhibitor of HIV-1. J. Virol. 2016, 90, 9495-9508. [CrossRef]

43. Pizzi, M. Sampling variation of the fifty percent end-point, determined by the Reed-Muench (Behrens) method. Hum. Biol. 1950, 22, 151-190. [PubMed]

44. Reed, L.J.; Muench, H. A simple method of estimating 50 percent endpoints. Am. J. Epidemiol. 1938, 27, 493-497. [CrossRef] 